\title{
III. Einige Beobachtungen über das Wachsthum der Kaliumaluminium-Alaunkrystalle.
}

\author{
Von
}

Z. Weyberg in Warschau.

(Mit 3 Textfiguren.)

\author{
Ex omnimoda exporientia, primum inventio causaram \\ et axiomatum verorum elicenda est; at lucifera ox- \\ perimentanon fractifera querenda. \\ Francisci Baconis „Novam Organum*. \\ Aphorismus LXX.
}

\section{§ 1. Einleitung.}

$\mathrm{Zu}$ den Messungen der Wachsthumsgeschwindigkeit der Krystallflächen des Eisenammoniumalauns ${ }^{1}$ ) waren Kaliumaluminium-Alaunkrystalle nöthig, welche keine Inclusionen enthielten, vollständig glatte Oberfläche besassen und die Flächen $\{110\}$ und $\{100\}$ so gross als müglich ausgebildet zeigten. Den Ideen des Prof. G. Wulff folgend ${ }^{2}$ ), erhielt ich Krystalle mit den gewünschten Eigenschaften nicht zufällig. Fine Reihe Züchtungen von Kaliumaluminium-Alaunkrystallen gab Veranlassung, einige Erscheinungen zu beobachten, die dazu dienen künnen, diejenigen Versuche G. Wulff's, welche die Morphologie des Krystallisationsprocesses aufklären, in einigen Details zu ergänzen.

Meine Versuche wurden in folgender Weise ausgeführt: Die gesättigte Lüsung wurde erwärmt und darin eine abgewogene Menge des übersättigenden Ueberschusses des Salzes aufgelüst. Die Krystallisation fand bei dersclben Temperatur, bei welcher die Lösung vorher gesättigt wurde, statt, also durch Abkühlen der übersättigten Lösung und nicht durch Verdunsten. In diese noch warme Lösung legte ich kleine homogene und glatte Alaunkryställchen, um auf ihnen den sich ausscheidenden übersättigenden Uebcrschuss sich absetzen zu lassen. Ijiese Kryställchen, in einer Zahl von

1) Diese Zeitschr. 34, 531.

2) Diese Zeitschr, 34, 449-530. 
6 bis 16, vertheilte ich gleichmässig auf den Boden des Gefässes; ich werde sie weiterhin »Keime nennen. Die Art und die Grösse dieser Keime werden in der Beschreibung jedes Versuches angegeben. Die Krystallisation fand in einem tiefen Keller eines alten Palastes bei constanter Temperatur statt ${ }^{1}$. Die Versuche dauerten 5 bis 35 Tage und wurden mit Quantitäten der Lösung von $\breve{b}$ bis 9,5 Liter in cylindrischen Gefässen ausgeführt, welche 30 oder 24 Centimeter Durchmesser des Bodens hatten. Die grüsste Anfangsübersättigung war $2,5 \%$, die kleinste $0,7 \%$. Die Uebersättigung der Lösung am Ende des Versuches wurde durch Wägen der erhaltenen Krystalle controlirt. Die Concentrationsströmungen beobachtete ich mit blossem Auge, mit Ausnahme von einem Falle. Das Salz, welches ich zu den Versuchen brauchte, wurde von der Firma C. A. F. Kahlbaum geliefert; das Wasser war destillirt.

\section{\$ 2. Beschreibung der Concentrationsströmungen.}

In Anbetracht der Wichtigkeit der Concentrationsströmungen ${ }^{2}$ ) werde ich meine Erklürung mit der Beschreibung dieser Erscheinung beginnen, wie ich sie speciell bei meinen Versuchen zu beobachten Gelegenheit hatte.

In $300 \mathrm{~g}$ gesättigter Lösung löste ich $3 \mathrm{~g}$ übersättigenden Ueberschusses und goss die Lösung dann in ein gläsernes kubisches Gefäss. Die Wände dieses Gefässes waren planparallel und sie zeigten im Toepler'schen Schlierenapparate vollständige optische Homogenität. In die Lösung, und zwar in der Mitte des Bodens des Gefässes, wurde ein weniger als zwei Millimeter im Durchschnitte messendes Kryställchen gelegt, welches mit seiner Fläche (TTT) die des Bodens berührte. Die Beobachtung geschah im Schlierenapparate mit der Linse von 2 m Brennweite, und der Krystall wurde mit einem Fernrohre beobachtet.

Im Beginn des Versuches erschien die Strömung als ein dünner glänzender Faden, welcher sehr langsam vom Centrum der Fläche (111) aufstieg. Am nächsten Tage bestand die Strümung deutlich aus vielen glünzenden faserigen Strömen, wobei die einzelnen Ströme von der Oberfläche des Krystalles abbogen und aufstiegen. Bei weiterem Wachsthume des Krystalles erhielt die Strömung eine steigende optische Schärfe und wurde fortwährend dicker und dicker. Später blieb sie ohne Veränderung bis zum 16. Tage des Versuches. An diesem wurde sie schwächer und fing an, ihre Schärfe zu verlieren, wobei sie sich fortwährend mehr und mehr

1) Zum Beispiel vom 12. Juli bis zum 15. October fiel die Temperatur von $+12, \mathbf{5}^{0}$ C. bis $+12^{0}$ C., vom 13 . October bis zum 6. November von $+12^{0} \mathrm{C}$. bis $+6^{0} \mathrm{C}$. (während letzterer Zeit wurde bei Frostwetter die Thür des Kellers geüflnet, uIn ihn abzukühlen;. Vom 6. November bis zum 1. März fiel die Temperatur von $+6^{0} \mathrm{C}$. bis $+5^{0} \mathrm{C}$.

2) S. die citirte Arbeit von G. Wulff. 
mit kleinen Strömen umgab; endlich am 22. Tage wurde die Strömung kürzer und stieg nicht mehr bis zur Oberfläche der Flüssigkeit empor. Nach 10 Tagen, von diesem Stadium an gerechnet, waren über dem Krystalle noch kleine und kurze Strümchen zu sehen, die über vielen Punkten der Oberll̈̈che des Krystalles ein wenig hervorstanden. Diese Ströme waren am 30. Tage schon nicht mehr zu sehen. Der Krystall wurde am 33. Tage herausgenommen und wog fast $3 \mathrm{~g}$.

In diesem Versuche wurde ein einzelner Krystall beobachtet, der in schwach übersättigter Lösung $(1 \%)$ wuchs. In jenen Versuchen, wo sich mehrere Keime in der Lösung befanden und die Uebersättigung stärker war, stellte sich der Gang der Erscheinung ein wenig anders dar.

Mehrmals wurden die Beobachtungen unter folgenden Umständen wiederholt: 31 Lüsung, Uebersättigung $130 \mathrm{~g}(2,5 \%), 10$ olktä̈drischc Keime von circa $5 \mathrm{~mm}$ im Durchmesser, Gefäss von $24 \mathrm{~cm}$ Bodendurchmesser, die llühe der Säule der Flüssigkeit $108 \mathrm{~mm}$. Der Gang der Erscheinung in diesen Versuchen war folgender:

Nach 24 Stunden vom Anfange des Versuches war über jedem Kryställchen ein dünnes langes glänzendes Bündel von Concentrationsströmen zu sehen, in welchem man keine Bewegung bemerken konnte. Am nüchsten Tage vergrösserten sich die Krystalle schon stark; dabei wurden die Bündel der Ströme viel breiter und schärfer, doch war die Bewegung der Flüssigkeit schwer zu bemerken. Am dritten Tage des Versuches bemerkte man noch einen stärkeren Zuwachs der Krystalle. In diesem Stadium ist schon fast die IFülfte des ursprünglichen übersättigenden Ueberschusses ausgeschieden worden, doch die Concentrationsströme sind noch stärker, als am vorigen Tage, und ihre Bewegung ist leicht zu bemerken. Im Laufe des vierten, fünften und sechsten Tages bemerkt man nur einen geringen $\mathrm{Zu}-$ wachs der Krystalle, und in den Strümen sieht man keinen Unterschied im Vergleiche damit, was am dritten Tage beobachtet worden war. Am siebenten Tage tritt eine augenscheinliche Abnahme der Thätigkeit der Concentrationsströme ein. Sie verlieren die optische Schärfe, die Bewegung nimmt allmählich ab, und am zehnten Tage stellen sie einen sich optisch schwach unterscheidenden Theil der Lüsung, in welchem sich keine Bewegung mehr bemerken lïsst, dar. Unterdessen wird auch dieser optische Unterschied allmählich schwächer, ihr Brechungscoefficient kommt mehr und mehr demjenigen der ganzen Lüsungsmasse gleich. So bleibt es einige Tage. Nach einer Woche ungeführ beginnt eine eigenthümliche Erscheinung. Die längere Zeit angewandte Lüsung des Kaliumaluminium-Alauns wird trübe infolge der Ausscheidung von basischem Aluminiumsulfat. Die trübende Substanz bleibt längere Zeit suspendirt, setzt sich aber allmählich auf dem Boden des Gefïsses ab, und bildet darauf eine recht dünne milchweisse Schicht. Solange die Strüme stark sind, nehmen sie die trübende 
Substanz mit, und sie ist dem Auge unsichtbar, mit $\Lambda$ usnahme jenes Theiles, welcher sich abgesetzt hat. Sobald aber die Strüme bis zum Minimum abgenommen haben, sind sie zu schwach, um die Substanz mit fortzureissen, sie lassen also hinter sich über den Krystallen eine schwebende Trübung zurück. Einige Tage nach dem Verschwinden der optischen Schärfe der Strüme bleiben noch über vielen P'unkten der Krystalle regungslos längliche Säulchen dieser Trübung stehen; sie ähneln dann dem sich über einem Iorfe erhebenden Rauche oder den über einer Wiese schwebenden Nebeln. Diese Trübung zeigt deutlich den Weg und die Richtung der abnehmenden Ströme: man sieht, wie sie, von der Fläche (111) sich abbiegend, von horizontaler lichtung eine verticale annehmen und wie sie sich von vielen Punkten der Oberfläche des Krystalles erheben, das heisst, dass zu dieser Zeit kein allgemeiner Strom vorhanden ist, der den ganzen Krystall abspült; man sieht, dass von den Kanten und den Ecken sich ein stürkerer Strom erhebt als von den Flüchen, denn über den Kanten und den Ecken stehen dichtere Nebel der Trübung. Ich bedauere sehr, dass die Umstände des Kellers, in welchem ich arbeitete, mir nicht erlaubten, diese interessante und schöne Erscheinung zu photographiren. Diese Nebel beobachtete ich in denjenigen Versuchen, welche genügend lange Zeit dauerten.

Wenn man in dasselbe Volum einer ebenso übersättigten Lüsung nicht zehn, sondern sieben Keime einlegt, so merkt man, dass die Zeit der $\mathrm{Zu}$ nahme der Ströme sich bedeutend verkürzt. Jeder Krystall erhält dann mehr übersättigenden Ueberschuss, die Krystalle nühren sich besser, erreichen schneller eine bedeutende Grüsse, verursachen immer stärkere Strüme, und dadurch erreicht man schneller die Verarmung der Lösung. In den Versuchen mit sieben Keimen bemerkte man schon am dritten Tage das Maximum der Thätigkeit der Ströme und am fünften Tage eine bedeutende Schwächung derselben.

$\Lambda \mathrm{ls}$ ich in die Lüsung 1,2 bis $0,7 \%$ des übersättigenden Ueberschusses einführte, war der Krystallisationsgang fast gleichmïssig: die Krystalle wurden jeden Tag unbemerkbar grösser, die Ströme waren sehr schwach, doch behielten sie ihre Kraft in demselben Grade lingere Zeit.

Aus den Angaben von G. Wulf $f^{1}$ ) über die Wachsthumsgrenze sieht man, dass die Concentrationsströme durchaus nicht nur ein unruhiges und regelloses Wachsthum des Krystalles begleiten, wie man es a priori denken künnte. Es muss aber in dem Krystallisationsprocesse immer ein solcher Moment eintreten, wo der Strom nicht mehr im Stande ist sich zu erheben, denn der Mangel an festen Material wird eher durch die Diffusion ausgeglichen, als es der dem Krystall anliegenden Schicht der Lüsung gelingt,

1) I. c. S. 462 . 
die zum Aufsteigen nöthige Geschwindigkeit zu erhalten. Das kann natürlich nur in sehr schwach übersättigter Lüsung stattfinden. Die Concentrationsströmungen müssen also selbst dạs langsamste und regelmässigste Wachsthum des Krystalles begleiten. $\Lambda \mathrm{ls}$ Beweis können alle meine Versuche dienen.

Viele von mir erhaltene Krystalle waren vollkommen homogen, doch waren die Ströme so lange über denselben $\mathrm{zu}$ bemerken, bis schon fast der ganze übersättigende Ueberschuss aus der Lüsung ausgeschieden war. So zum Beispiel in einem von den vorher beschriebenen Versuchen (5 1 lüssung, $130 \mathrm{~g}$ Uebersättigung) wurden die Krystalle herausgenommen einen Tag nachdem die Ströme aufhörten sichtbar zu werden. Sie wogen $128 \mathrm{~g}$. In den letzten Momenten des Versuches also war in der Lösung eine Uebersättigung von $2 \mathrm{~g}$, das heisst $0,04 \%$, und dieser geringen Uebersättigung ungeachtet bemerkte man die Ströme noch am Tage vorher.

Ich unterbrach den grüssten Theil meiner Versuche dann, wenn die Ströme deutlich zu sehen waren, doch dem Gewichte der Krystalle nach blieb die Lösung sehr schwach übersättigt. Nach der Unterbrechung des Versuches zeigte sich in der umgerührten Lösung auf dem Boden des Gefässes erst nach einem oder zwei Tagen eine dünne Schicht von winzigsten Krystallen.

Das ist der Beweis dafür, dass das Wachsthum der Krystalle vermittelst der Diffusion allein eine ausnahmsweise Erscheinung ist.

\section{\$ 3. Concentrationsströme als Ursache der Erscheinung der Vicinalfächen.}

Auf den Flächen \{111\} des Kaliumaluminium-Alauns bemerkt man fast immer drei Vicinalflächen, welche gleichsam die Flïche eines Triakisoktaëders darstellen, dessen Indices sich sehr wenig von einander unterscheiden.

Nach der Ansicht von G. Wulf ${ }^{1}$ ) besteht die Ursache der Entstehung der Vicinalflächen in dem Einflusse der Concentrationsstrümungen, weil die Lage der Krystallmolekeln nicht nur von der Richtkraft der Krystallisation, sondern auch von derjenigen der Concentrationsstrümungen abhüngen muss.

Die Strüme, welche den Krystall umspülen und gegen die Flïche (111) eines mit (TTT) aufliegenden Alaunoktaëders ansteigen, bewegen sich zuerst längs derselhen, biegen aber dann von dieser Fläche ab und steigen empor. In Folge der dreieckigen Form der oktaëdrischen Fläche bilden diese Ströme drei einzelne Gruppen: jede Gruppe tritt von einer Kante aus auf die Fläche ein und verliert hier ihre ursprüngliche Geschwindigkeit, da sie sowohl nach dem Centrum der Flïche als auch nach oben strebt. Alle drei Gruppen vereinigen sich endlich über dem Centrum der Fliiche

\footnotetext{
1) I. c. S. 461 .
} 
und verlassen den Krystall. Jede dieser Gruppen von Strömen führt die Molekeln der entsprechenden dreieckigen Partie der Oktaëderfläche also aus ihrer normalen Stellung; als Resultat erscheinen auf der Fläche (111) drei Vicinalflächen, welche Triakisoktaëderflächen ähnlich sind.

Dass die Strüme längs der Fläche gleiten und sich gleichzeitig von ihr, je nach der Annäherung zum Centrum dieser Fläche, abbiegen, kann man unmittelbar bemerken.

Als Beweis dafür, dass die Ströme, welche die Fläche (111) umspülen, drei einzelne Gruppen bilden, kann Folgendes dienen.

In einer grossen Masse der Lösung (von $\breve{b}$ bis $9,5 \mathrm{l}$ ), mit welcher ich gewöhnlich arbeitete, konnte man trotz sorgfaltiger Filtrirung die Anwesenheit einer bedeutenden Menge von Staub, Fasern vom Handtuche, mit welchem man das Gefäss reinigte, vom Filtrirpapier u. s. w. nicht vermeiden. Ausserdem befand sich in der Alaunlösung die vorher besprochene Trübung. Alle diese Körper setzen sich bei ruhigem Zustande der Flüssigkeit auf dem Boden des Gefässes und auf der Oberflüche der im Gefüsse wachsenden Krystalle ab. Auf der Fläche (111) der oktaëdrischen Krystalle jedoch zeigt sich dieser Niederschlag nicht gleichmässig: er bildet drei geradlinige, nach der Mitte hin stärker werdende Streifen, welche im Centrum der Fläche zusammentreffen und einen kleinen Hügel bilden. Diese Streifen liegen in der Richtung der Triakisoktaëderkanten, d. h. sie sind auf dieselbe Weise wie die Kanten der Vicinalflächen orientirt. Selhstverständlich wurden die sie bildenden Theilchen durch die Concentrationsströme nach der Stelle, wo die Geschwindigkeit der Strüme am geringsten war, d. h. dahin, wo zwei einzelne Stromgruppen zusammenstossen, hingespült. Die eben beschriebene Lage dieses Niederschlages erreicht den höchsten Grad ihrer legelmässigkeit, wenn die Ströme das Maximum ihrer Stärke erreicht haben. Später, wenn die Ströme schwächer werden, können sie nicht mehr den Krystall ganz umspülen, und sie erheben sich nicht vom Centrum der Fläche (111), sondern von vielen Punkten der Oberfläche des Krystalles; die beschriebene Figur verwischt sich dann, ihre Aeste werden von den einzelnen Strömen vertilgt, und dabei verschwinden auch die Vicinalflächen. So zeigten von zehn Krystallen dieser Versuche (fünf Liter der Lüsung, Uebersättigung. $2,5 \%$, Diameter des Gefüsses $24 \mathrm{~cm}$, zehn kleine Keime, die Lüsung nach der Unterbrechung des Versuches fast ohne Uebersättigung), welche 31 Tage dauerten, bloss drei Krystalle die Vicinalflïchen, und auch diese Flächen waren schwer zu bemerken. Im Gegentheil waren die Vicinalffächen sehr deutlich bei denjenigen Krystallen, welche sich in stark übersättigter Lüsung bildeten, oder welche aus schwach übersättigter Lüsung während der vollen Thätigkeit der Ströme herausgenommen waren. Gleichzeitig mit dem Zuwachs der Krystalle entwickeln sich immer mehr und mehr die Würfelflächen, welche die Ecken der drei- 
eckigen Fläche des Oktaëders abstumpfen. Die Ströme theilen dann jeden Streifen des Niederschlags in zwei. Die Kanten der Vicinalflächen krümmen sich wellenförmig und werden unterbrochen.

Bei einer schwachen Uebersättigung erscheinen die Concentrationsströme als ein dünnes Bündel, welches sich vom Centrum der Fläche (111) erhebt. Auf dieser Fläche erscheint dann bloss eine einzige Gruppe von Vicinalflächen vollständig symmetrisch orientirt. Wenn aber die Uebersättigung der Lüsung bedeutend ist, so bemerken wir über dem ganzen Krystalle viele glänzende Strüme. Dann sieht man auf der Fläche (111) mehrere Gruppen von Vicinalflächen. Manchmal verschiebt sich der dünne Concentrationsstrom (welcher in schwach übersättigter Lüsung erscheint) zur Seite, dann wird auch die Gruppe der Vicinalflächen verschoben.

\section{$\S$ 4. Wirkung der Concentrationsströme auf den Charakter der Krystalloberflächen.}

Jene Krystalle, welche in stark übersättigter Lösung (gegen 10\%) wuchsen, zeigen auf der Fläche (111), d. h. auf derjenigen Oktaëderfläche,

Fig. 1.

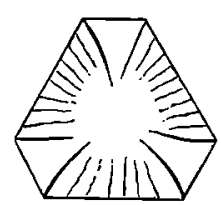
welche der Oberfläche der Lüsung zugekehrt ist, lisse, deren Anordnung Fig. 1 zeigt. Dieselben haben die gleiche Richtung wie die Ströme und können nur durch deren Wirkung erklärt werden. Gleichzeitig mit der Abnahme der Uebersätligüng werden die Risse immer schwächer, endlich verschwinden sie und statt derselben erscheinen die Vicinalflächen.

Auf denjenigen Flächen, welche einen Winkel mit dem Boden des Gefässes bilden, bemerken wir auf den Alaunkrystallen eine Streifung. Diese Streifen erscheinen entweder parallel, oder senkrecht zur Richtung der Concentrationsströme. Die Streifen der ersten Art zeichnen sich durch ihre Regelmässigkeit aus; man kann sie, wie es mir scheint, mit den beschriebenen Rissen vergleichen: sie erscheinen bei starker Uebersättigung, die Streifen der anderen Art in Gegentheil bei schwacher.

Die letztere Streifung (senkrecht zur Concentrationsströmung) ist viel unregelmässiger. Man darf sie dann als Resultat des Mangels an Uebersättigung betrachten, wenn die Uebersättigung zu unbedeutend ist, um die ganze Oberfläche des Krystalles zu ernähren. Die Fläche (T11) und die ihr entsprechenden haben über sich die Flächen des Dodekaëders; solche Flächen, wie (T11), erscheinen immer in der Nähe der Dodekaëderfläche wie angefressen, denn die Ströme sind nicht im Stande sie genügend zu bespülen; diese fliessen theils nach oben fort, theils werden sie durch die viel energischer wachsende Dodekaëderfläche angezogen, so dass für solche Flächen, wie (T11), der Stoff nicht ausreicht ${ }^{1}$ ).

1) I. c. S. 629 . 
Das beschriebene Anfressen der Flächen war besonders an demjenigen Krystalle gut zu sehen, auf welchem man die Ströme im Schlierenapparate bcobachtete. Dieser Versuch, bei sehr unbedeutender Uebersättigung ( $1 \%$ \% und kleiner Menge der Lösung (300 g), dauerte mehr als 30 Tage, und der ühersättigende Ueberschuss wurde fast vollständig ausgeschieden, da der Krystall fast $3 \mathrm{~g}$ wog. Alle oktaëdrischen Flächen dieses Krystalles, welche mit dem Boden des Krystallisirgefüsses einen stumpfen Winkel bildeten, waren unvollkommen ausgebildet, und im Allgemeinen mit einer dem Boden des Gefüsses parallelen Streifung bedeckt. Solche horizontale Streifung und Unvollkommenheit war aber bloss bei denjenigen Flächen zu bemerken, welche mit dem Boden des Gefüsses einen stumpfen Winkel hildeten, denn von solchen Flächen steigt der Strom sehr leicht nach oben. l)ie Verticalflächen und diejenigen, welche mit dem Boden einen spitzen Winkel bildeten, waren dagegen, selbst bei der unbedeutendsten Uebersättigung, vollkommen ausgebildet, denn ihre Lage erlaubte ihnen von den Strömen vollstïndig abgespült zu werden.

\section{$\$$ 5. Einfluss der Concentrationsströme auf den Habitus der Krystalle.}

Jer liabitus der Krystalle ist ein Resultat ihrer Orientirung gegen die Concentrationsströme ${ }^{1}$ ), d. h. er hängt davon ab, mit welcher Fläche der Krystall den Boden des Gefüsses berührt. Die Frage, weshalb der Krystall auf dieser oder jener Fläche liegt, ist fast ungelüst.

Die Kaliumaluminium-Alaunkrystalle, die sich aus ihrer reinen wässerigen Lösung ausscheiden, liegen auf dem Boden des Krystallisationsgefässes so, dass sie meistentheils den Boden mit der Oktaëderfläche berühren; viel seltener liegen sie auf der Würfelfläche; und diejenigen Krystalle, welche auf der Dodekaëderfläche liegen, stellen eine ausserordentliche Seltenheit dar. Unter mehreren hunderten der von mir erhaltenen Nlaunkrystalle lagen bloss sechs Krystalle auf (110). l)ie Alaunkrystalle liegen also am häufigsten auf denjenigen Flüchen, deren Wachsthumsgeschwindigkeit am geringsten ist, und am seltensten auf jenen Flächen, deren Wachsthumsgeschwindigkeit am grüssten ist 2). An denjenigen Krystallen, welche auf der Oktaëderfläche liegen, künnen die $\{100\}$ und $\{110\}$ eine bedeutende Breite erreichen. Auf dem Würfel und dem loodekaëder liegend gewachsene Krystalle sind $\{100\}$ und $\{110\}$ dem oktaëder untergeordnet.

Wenn man den Alaun aus stark übersättigter Iüsung oder aus einer kleinen Menge der Flüssigkeit krystallisirt, oder wenn die Krystallisation

1) 1. c. S. 463,464 .

घ) Die Wachsthumsgeschwindigkeit der Alaunkrystallflächen in ihrer reinen Lösung $=V_{111}<V_{100}<V_{110}$. 
in einem Laboratorium stattfindet, wo häufige kleine Erschütterungen der Krystallisirgefässe durch Hin- und Hergehen von Personen hervorgebracht werden, oder wenn in schwach übersättigter Lösung die Krystallisation durch Berühren der Oberfläche der Lösung mit einer unreinen Nadel hervorgerufen wird, so erhält man sehr selten solche Kryställchen, die auf $\{100\}$ liegen und nie auf $\{110\}$ liegende; sie liegen fast immer auf $\{111\}$. Während aus einer grossen Menge der Lösung auf den Keimen langsam grosse Krystalle erwachsen, erscheinen ausser diesen selbständig schon am vierten Tage, manchmal auch später (vom Anfange des Versuches gerechnet), auf dem Boden des Gefässes einige kleine Kryställchen, welche gewöhnlich sehr vollkommen sind und sehr langsam wachsen. Unter diesen Krystallen fand ich sechs solche, die auf der Dodekaëderfläche lagen. Dabei stellen die auf $\{100\}$ liegenden Krystalle unter diesen Krystallen keine Seltenheit dar, obgleich sie in einer kleineren Menge als die auf dem Oktaëder liegenden erscheinen.

Die Umstände der Erscheinung dieser selbständigen Krystalle stellen augenscheinlich nichts Besonderes dar. Vielleicht könnte man hier nur die Abhängigkeit der Entstehung solcher seltenen Habitus von der relativen Schwierigkeit der Ausscheidung der Uebersättigungsreste erblicken.

Sobald nun ein Krystall auf der angegebenen Fläche liegend erscheint, bekommt er unter dem Einflusse der Concentrationsströme eine besondere Form, welche immer dem auf der angegebenen Fläche liegenden Krystalle eigenthümlich ist.

In schwach übersättigten Lüsungen (unter 1,3\%) erscheinen die Krystalle jedes Ilabitus ziemlich nahe dem theoretischen Oktaëder, denn unter diesen Umständen existirt nur eine geringe Wirkung der Concentrationsströme, welche den Krystall bespülen. In den Lösungen mit der Uebersättigung gegen $2 \%$ und mehr erhalten die auf $\{111\}$ liegenden Krystalle die Form einer dicken Tafel; in den auf $\{100\}$ liegenden Krystallen entwickelt sich bloss ihre obere Hälfte; diejenigen, welche den Boden mit $\{110\}$ berühren, erhalten eine längliche domatische Form. (Erklärung siehe G. Wulff, l. c. S. $463-464$.)

\section{§ 6. Einfluss der Concentrationsströme auf die relativen Dimensionen der Flächen.}

In der Einleitung deutete ich an, dass für mich die Gewinnung von Krystallen mit möglichst breiten Flächen $\{110\}$ und $\{100\}$ von grosser Bedeutung war.

Von den Kaliumaluminium-Alaunkrystallen, welche man aus reiner wässeriger Lösung erhält, wissen wir, dass $\{100\}$ und $\{110\}$ untergeordnet sind, d. h. dass die Breite dieser Flïchen im Vergleich mit denen von $\{111\}$ unbedeutend ist. Ohne irgend welche künstliche Methoden zu verwenden, 
sondern nur durch das Aussuchen der entsprechenden Bedingungen erhielt ich Alaunkrystalle mit sieben bis acht Millimeter breiten, dodekaëdrischen Flächen bei einer Breite der oktaëdrischen Flächen von zehn Millimeter und weniger.

Auf Grund der Versuche von Hrn. G. Wulff über die Krystallisation des Salzes $\left(\mathrm{NH}_{4}\right)_{2} \mathrm{Z} / \mathrm{m}\left(\mathrm{SO}_{4}\right)_{2} \cdot 6 \mathrm{H}_{2} \mathrm{O}$ kann man Folgendes schliessen: Wenn sich eine kleine Zahl von wachsenden Krystallen auf dem Boden des Krystallisationsgefässes befindet, so bringen die Concentrationsstrüme eine genügende Menge des aus der Lösung sich ausscheidenden Salzes zu den Krystallen; dabei erscheinen dic abstumpfenden Flächen entweder gar nicht, oder sie sind sehr schmal. Bei einer grösseren Anzahl von Krystallen tritt jedoch ein Mangel an Uebersättigung ein; infolge dessen können die Concentrationsströme auf den lianten des Krystalles keinen Ueberschuss absetzen, und deswegen crscheinen statt der Kanten breite abstumpfende Flächen.

Hr. G. Wulff gieht drei photographische Aufnahmen'), welche uns zeigen »wie während des Wachsthums je nach der Annäherung der Ecke eines Krystalles an einen anderen wachsenden Krystall die die Ecke ahstumpfende Flïche crscheint und wächst«. Diese von Hrn. (i. Wulff für mikroskopische Krystalle beschriebene Erscheinung hatte ich aber Gelegenheit mehrere Male im sehr grossen Maassstabe zu beobachten. Sehr häufig entstand gegenüber der Würfelfläche der Seitenzone des auf (T00) liegenden Krystalles ein ebenso orientirter Krystall. Beim Weiterwachsen dieser Krystalle blieb zwischen ihnen ein breiter /wwischenraum, denn die Stoffzufuhr zu den Würfelfliichen (welche diesen Zwischenraum begrenzten) war sehr unbedeutend, weil der kleine Krystall den sich ausscheidenden Ueberschuss den ihm zugekchrten Theile des grossen entzog und dadurch ein Zurückbleiben seiner Würfelfläche bewirkte. I)iese beiden Flächen erscheinen daher sehr gross im Vergleich mit den anderen Würfelflächen an diesen Krystallen. Ein analoges Beispiel bot ein dodekaëdrischer Krystall dar, der in 9,5 I Jüsung zusammen mit sechs anderen Keimen gezüchtet wurde und sich auf ein Drittel des Radius des Gefässes von der Wand entfernt befand (von der Wand fünf Centimeter, vom Centrum zehn Centimeter; er lag so, dass seine binäre Symmetrieaxe parallel zum Radius des Gefässes war). Jenes Linde des Krystalles, welches gegen die Wand des Gefässes gerichtet war, hatte eine breite Würfelfläche $(1 \mathrm{qcm})$; die andere Ecke aber, zum Centrum des Gefässes gerichtet, hatte bloss eine unbedeutende Abstumpfung (4 qmm). Wenn man also zwei Krystalle neben

1) Die citirten photographischen Aufnahmen und deren Erläuterung sind in der russischen Ausgabe der Abhandlung von H. G. Wulff enthalten. In der deutschen sind sie ausgelassen worden.

Groth, Zeitschrift f. Krystallogr. XXXVl. 
einander ziemlich nahe mit den entsprechenden Flächen stellt, so kann man breite Abstumpfungen erhalten; das crreicht man aber nur dann, wenn die angegebenen Flächen vertical gestellt werden können. Die dodekaëdrischen Flächen kann man nicht auf diese Weise behandeln, da sie einen ziemlich stumpfen Winkel mit dem Horizonte bilden; dabei kann man aber auch leicht, in Folge eines Mangels an Uebersüttigung, skelettartige Flïchen erhalten. In diesem Falle ist es nöthig, dass alle im Krystallisirgefiisse wachsenden Krystalle einander die Uebersättigung entziehen. Dies erreicht man durch Züchten einer genügenden Zahl von Keimen in einer ziemlich bedeutend übersättigten Lüsung. In vielen Versuchen wurden auf fünf Liter vier bis sieben Keime genommen, und ungeachtet der verschiedenen Procente der Uebersättigung erhielt man schmale granatoëdrische Flächen. Doch die Menge der Keime und der Grad der Uebersättigung sind nicht die einzigen Bedingungen. In ein Gefäss von $30 \mathrm{~cm}$ Durchmesser wurden neun Liter der Lüsung mit 2\% Uebersättigung eingegossen, welche eine Säule von $135 \mathrm{~mm}$ Höhe bildeten; darin legte man 14 Keime ein. In fünf Liter ebenso übersättigter Lösung im gleichen Gefässe $(76 \mathrm{~mm}$ hohe Säule) wurden 1/ Keime hineingelegt. Diese beiden Versuche gaben Krystalle mit sehr schmalen $\{110\}$. Die breitesten Flïchen $\{110\}$ (bis $8 \mathrm{~mm}$ ) erhielt ich in einer $108 \mathrm{~mm}$ hohen Lösungsschicht (5 l, Uehersättigung 2,5\% $\% 10$ Keime, $24 \mathrm{~cm}$ Durchmesser des Bodens des Gefässes). Die analogen Versuche in einer Lüsungsschicht, welche dicker oder dünner als $108 \mathrm{~mm}$ war, bieten jedoch immer nur schmale dodekaëdrische Flächen dar. Hier spielt also die IIöhe der Flüssigkeitssäule eine wichtige Rolle.

Man könnte vermuthen, dass von der Dicke der Lösungsschicht die Geschwindigkeit der Circulationsströme und die Erneuerung der die wachsenden Krystalle unmittelbar umhüllenden Lösung abhüngt. Das Optimum zur Erscheinung der breitesten Fliichen $\{110\}$ findet bloss bei einer gewissen Dicke der Lösungsschicht statt.

Wenn die aufsteigenden Strüme gerade entsprechend verminderte Geschwindigkeit besitzen, und dabei auch genug Krystalle vorhanden sind, sodass sie von der zufliessenden Uebersättigung nicht zu reichlich ernährt werden, so kommt das zu Stande, was wir in allen Krystallisationen in fünf Liter mit der Uebersättigung $2,5 \%$ und mit zehn Keimen in einem Gefässe von $24 \mathrm{~cm}$ Durchmesser, d. h. in einer Süule der Flüssigkeit von $108 \mathrm{~mm}$, gesehen haben. Dic niedersteigenden Ströme gelangen zu den Krystallen ziemlich stark übersättigt; sie werden leichter, nachdem sie zum Theil ihre Uebersättigung auf den niederen Partien des Krystalles gelassen haben; sie erheben sich auf die Flächen $\{110\}$, von wo sie theils nach oben, theils auf die Fläche (111) steigen; das tritt jedoch nicht sehr rasch ein, infolge dessen fliessen dic Ströme mit neuer Uebersättigung ziemlich langsam. Bei diesem Processe streben die Ströme die nährende Oberfläche 
des Krystalles möglichst zu vermindern ${ }^{1}$ ), so dass bei zehn ziemlich nahe bei einander liegenden Krystallen ein Mangel an sich ausscheidendem Ueberschusse bei verminderter $\Lambda$ bgabe desselben stattfindet. Die Ströme setzen deswegen den Stoff nicht auf den Kanten $\mathbf{a b}$, sondern sie bilden an deren Stelle breite abstumpfende Flächen. Dafür sprechen die folgenden Gründe: 1) die breiten Abstumpfungen erscheinen bloss an denjenigen Krystallen, die den Boden des Gefässes mit der Fläche ( $\overline{1} T \bar{T}$ ) berühren, und nur in den oberen, nicht aber an den Seiten solcher Krystalle; deren Seitenflächen $\{110\}$ sind senkrecht zum Boden des Gefässes, deswegen gleitet der Strom längs dieser Flächen, ernährt sie reichlich und kann sie nicht verlassen; dabei nähren sich die unteren Partien des Krystalles viel besser als die oberen; 2) infolge dessen entstehen schmale Abstumpfungen an den Krystallen, welche auf den Flächen $\{100\}$ oder $\{110\}$ liegend wachsen, unter recht verschiedenen Umstïnden der Krystallisation.

Dic unmittelbare Beobachtung der Entwickelung der Dodekaëder- und Würfelflächen begründet diese $\Lambda$ nschauungen. In den angegebenen FünfLiter-Versuchen erreichte die Breite der abstumpfenden Flächen das beobachtete Maximum. In diesen Versuchen besassen die Concentrationsströme schon am dritten Tage die volle Kraft, die Krystalle waren zu dieser Zeit ziemlich gross und die Dodekaëderflächen hatten fast dieselbe Breite, welche am Ende des Versuches beobachtet wurde. Diese Flächen entwickeln sich nämlich während der vollen Thätigkeit der Concentrationsströme, und wenn die letzteren verschwinden, so vermindert sich ihre Grösse im Vergleich mit der ganzen Oberfläche des Krystalles, weil sie ihre ursprüngliche Grösse behalten ${ }^{2}$ ); ausserdem vermindern diese Flächen auch absolut ihre Grösse, indem sie immer schmäler werden. Die weniger energisch, d. h. langsamer wachsenden Würfelflächen befinden sich unter günstigeren Umständen, als die von $\{110\}$; sie sind immer breiter als die letzteren; wenn sich also $\{110\}$ entwickelt, so kann das bei $\{100\}$ noch besser der Fall sein.

So sind die Verhältnisse bei der entsprechenden Uebersättigung der Lösung.

Bei einer zu geringen Uebersättigung findet die Krystallisation langsam statt. Die Ströme erhalten die Möglichkeit, ihre ganze Uebersättigung an dic Krystalle abzugeben, und dadurch kommt es nicht zu einem Mangel, welcher durchaus zur Bildung der breiten Abstumpfungen nöthig ist.

Breite Abstumpfungen bilden sich dann, wenn die Ströme den ganzen Krystall umspülen, die Ausscheidung des übersättigenden Ueberschusses schnell stattfindet und wenn dieser Ueberschuss in kurzer Zeit zwischen

1) Wulff 1. c. S. 460 .

2) Als Beweis, dass die Flächen $\{110\}$ während des Wachsens einige Zeit von derselben Breite bleiben, dient die Anordnung, in welcher sich die unter diesen Flächen liegenden Einschlüsse befinden. 
mehreren Krystallen getheilt werden muss. Diese Umstände fanden in meinen Fünf-Iiter-Versuchen mit der Uebersiittigung $2,3 \%$ und zehn Keimen gegen den dritten Tag des versuches statt.

Unter solchen Umständen nährt sich der Krystall ausnahmsweise bloss von den Strömen, denn die Diffusion durch die den Krystall bedeckende Membran eines sich schnell bewegenden Stromes ist unbedeutend. Nach den Worten G. Wulff's strcben zu dieser Zeit die Ströme den Krystall abzurunden « ${ }^{1}$ ). Je nach der Verminderung der Uebersättigung der Lösung fangen die Ströme an schwächer zu werden, bleiben in Folge kleiner Geschwindigkeit eine längere Zeit bei dem Krystalle und nähren dadurch reichlich die energischeren Fliichen. Inzwischen hören sie auf, eine allgemeine, den Krystall umspülende Strömung zu bilden und zerfallen in mehrere kleine Einzelstrüme, welche sich von vielen Punkten der Krystalloberfläche erheben. Dic Jiffusion wirkt dann mit den Strömen zusammen auf das Wachsthum des Krystalles; die wenig energischen Oktaëderflächen hören fast vollständig auf zu wachsen, während die Würfel- und Dodekaëderflächen gut genährt werden, und die vorher beschriebene Trübung zeigt recht deutlich, wie letztere stärkere Ströme hervorrufen, also im Vergleich mit den Oktaëderflächen stärker wachsen. Als Resultat ergiebt sich Folgendes: In allen Versuchen mit kleiner ursprünglicher Uebersättigung $(1,3 \%, 1,2 \%, 1 \%, 0,7 \%)$ zeigten meistentheils die Krystallc keine Flächen $\{110\}$ und nur einige darunter sie in Form von sehr schmalen Abstumpfungen. Wenn aber fünf Liter Lösung mit $2,5 \%$ oder $2 \%$ Uebersättigung mit zehn Keimen in einem Gefässe von $24 \mathrm{~cm}$ Durchmesser (Flüssigkeitssäule $108 \mathrm{~mm}$ ) 20 oder sogar 30 Tage stehen gelassen wurden, sodass fast die ganze Vebersättigung sich ausschied, so waren die Flächen $\{110\}$ anfangs recht breit, dann wurden sie allmählich schmäler, und am Ende des Versuches hatten darunter bloss einige recht seltene eine Breite von sechs Milimeter, nämlich diejenigen, welche anfangs so breit waren, dass ein Zuwachsen nicht mehr möglich war, weil der übersättigende Ueberschuss ausgeschieden und daher kein Stoff dafür vorhanden war.

Was diesen "Mangel * der Ernährung anbetrifft, von welchem mehrere Male gesprochen wurde, so zeigt der folgende Versuch, dass dieser Mangel durchaus nothwendig zur Entstehung der breiten $\Lambda$ bstumpfungen ist. In fünf Liter Lösung (Uebersättigung $2,50 \%$, Gefäss von $24 \mathrm{~cm}$ Durchmesser, Säule $108 \mathrm{~mm}$ ) wurden statt zehn bloss sieben Keime gelegt. $\Lambda \mathbf{n}$ den Krystallen dieses Versuches war während ihres Wachsens keine einzige Fläche weder des Würfels, noch des Dodekaëders breiter als ca. vier Millimeter zu sehen, und am 18. Tage waren schon fast alle Flächen $\{110\}$ verwachsen.

1) l. c. S. 460 . 
Ausser dem, was bisher gesagt wurde, ist zum Auftreten der breiten Ibstumpfungen bei einer gewissen Uebersättigung der Lüsung und Höhe ihrer Süule noch eine bestimmte Grösse des lirystalles nothwendig. Sobald ich zu Keimen Kryställchen von ca. zwei Millimeter Durchmesser oder grüssere als zehn Millimeter nahm, erhielt ich dic breiten Flächen $\{110\}$ nicht, obgleich alle anderen Bedingungen erlüllt wurden. Die breitesten Flächen $\{110\}$ erhielt ich dann, wenn ich in fünf Liter (Uebersütligung 2,3\%, Gefïss von $24 \mathrm{~cm}$ Durchmesser) zehn Kcime legte von ganz gleicher (irüsse (von fünf Millimeter Durchmesser), die vollstïndig durchsichtig, mit glatten Oberfliichen, waren. Grosse Keime rufen sogleich starke Concentrationsstrüme hervor, die Krystallisation tritt schnell und ungleichmïssig ein, die Erneuerung der Iüssung um den Krystall herum findet sehr rasch statt, und ein Mangel der Nahrung kommt nicht zu Stande. Je kleiner der Krystall ist, desto schwïcher sind die von ihm hervorgerufenen Ströme und umgekehrt. Dic breiten $\Lambda$ bstumpfungen erscheinen bloss bei einer gewissen Geschwindigkeit des Krystillisationsprocesses und bei ciner gewissen Grüsse des Krystalles.

Die Entwickelung also der breiten Abstumpfungen ist daher mit folgenden Umstïnden verbunden: 1) mit der Entfernung der Krystalle von einander, 2) mit ihrer Zahl, 3) mil ihrer Grüsse, 4) mit der Orientirung der Krystalle gegen die Concentrationsströme, 5) mit dem (irade der Uebersättigung der Lüsung und 6) mit der Djeke der Lüsungssschicht.

\section{\$7. Zusanmenhang zwischen den Concentrationsströmen und der Entstehung von Einschlüssen im Krystalle.}

Bei der Beobachtung eines wachsenden Alaunkrystalles, -der den Boden des Gefässes mit der Fläche (00T) berührt, kann man leicht sehen, dass von der Spitze des Oktaëders, welche durch die Fläche (001) abgestumpft wird, ein Strom, welcher sichtbar den Krystall umlüllt, aufsteigt. Von irgend welchem Punkte der Combinationskante $\{100\}:\{111\}$ erhebt sich cin Strom, der nach innen gekrümmt ist. Nachdem dieser Wirbel auf $1-2 \mathrm{~mm}$ über den Krystall aufgestiegen ist, verliert er seine Wirbelbewegung und kann von dem übrigen Bündel der geraden Ströme nicht mehr unterschieden werden. Diese Erscheinung wiederholt sich periodisch. Solche Wirbel erscheinen bloss auf den Kanten dort, wo zwei Flächen, deren Wachsthumsgeschwindigkeit verschieden ist, zusammenstossen. Jede von dicsen Flächen entnimmt dem Strome verschiedene Mengen der Uebersättigung, wodurch auf der Kante eine sichtbare Perturbation der IIomogenität des Stromes entsteht.

Viele solche Krystalle, die auf der Flïche (00T) lagen, erhielt ich fast durchsichtig; bloss vom Anfangspunkte des Wachsthumes verlief zur Fliche (001) eine schwach sichtbare, zarte milchweisse Pyramide von Einschlüssen. 
Von einem derartigen Krystalle schliff ich eine Platte parallel zu (001), um den Querschnitt des Anwachskegels zu erhalten. In diesem Schliffe zeigte sich dem blossem Auge ein weisslich trübes Quadrat, dessen Kante gegen

Fig. 2.

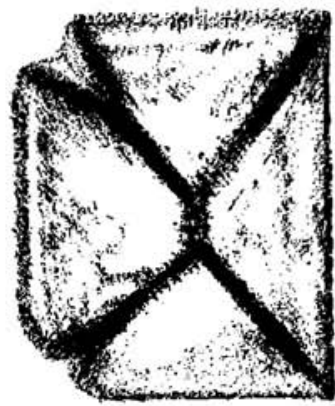
$3 \mathrm{~mm}$ hatte, wie es in Fig. 2 in 13 facher Linearvergrösserung dargestellt ist ${ }^{1}$ ). Seine Kanten sind parallel zu den Combinationskanten des Würfels und Oktaëders, und die Reihen der Einschlüsse in den Diagonalbändern sind parallel zu den Com-

Fig. 3.

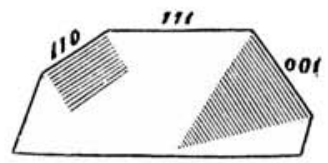
binationskanten des Würfels und des Rhombendodekaëders. Hier sind also die Einschlüsse ganz einfach die Spuren der Flïchen, welche sich vom Centrum des Krystalles während seines Wachsens entfernen. Dasselbe zeigt Fig. 3, welche schemalisch die Anordnung der Einschlüsse in den weniger vollkommenen Krystallen, welche während des Wachsens auf der Fliche (i $\bar{T} \bar{T}$ ) liegen, darstellt.

Wenn wir also in den Strömen Perturbationen über den Combinationskanten und in den Krystallen unter diesen Kanten Einschlüsse sehen, so sind wir im Stande, mit grosser Wahrscheinlichkeit Folgendes zu behaupten: Die Perturbationen in den Strömen auf den Grenzen der Flächen, welche verschiedene Wachsthumsgeschwindigkeit besitzen, rufen die Entstehung der Einschlüsse im Krystalle hervor.

Zu Fig. 2 ist zu bemerken, dass den allerenergischsten Flächen $\{110\}$ die grössten Einschlüsse entsprechen.

Was die relative Geschwindigkeit der sich vom Würfel und Oktaëder erhebenden Strüme anbetrifft, so beobachtet man unmittelbar dic grössere Geschwindigkeit derselben auf der Würfelfläche, was mit den Forderungen der Theorie vollkommen übereinstimmt.

In den rotirenden Krystallisationsgefässen ${ }^{2}$ ) waren die Ströme sehr klar zu sehen. Sie steigen die ganze Zeit hindurch senkrecht nach oben, und der Krystall wechselt fortwährend seine Orientirung gegen dieselben. Wenn die Würfelflïche nicht horizontal ist, so erheben sich die Ströme von der Oktaëderfläche, sie sind dann kaum zu sehen. Sobald aber die Würfelfläche wieder horizontal wird, werden auch die Strüme recht scharf

1) Die Ausführung dieser Photographie habe ich der Liebenswürdigkeit von Prof. G. Wulff zu verdanken. Ich benutze die Gelegenheit, um ihm meinen besten Dank für die Initiative und werthvolle Hülfe bei meinen Versuchen auszusprechen.

2) Ueber die Krystallisation in den rotirenden Gefässen siehe G. Wulff I. c. S. 502 und 508 . 
und eine Acceleration ihrer nach oben steigenden Bewegung ist zu bemerken.

Jetzt werde ich einige Worte über die Umstände, unter welchen man Krystalle ohne Einschlüsse erhält, sagen.

Herr G. Wulff bemerkt, dass der Krystall dann mit Einschlüssen überfüllt ist, wenn er als liesultat der Thätigkeit energischer Concentrationsstrüme erscheint, d. h. wenn er in stark übersitttigter Lösung wächst 1 ). Daraus künnte man a priori das Umgekchrte schliessen, nämlich: dass die in schwach übersiittigter Lüsung gewachsenen Krystalle keine Einschlüsse besitzen, und, je schwächer die Lösung übersüttigt ist, desto grössere Garantie vorhanden wäre, dass wir in solcher Lüsung fast vollständig durchsichtige Krystalle erhalten. Im Allgemeinen findel dies auch statt; auf die relative Durchsichtigkeit der Krystalle haben jedoch ausser dem Grade der Uebersüttigung der Lösung viele andere Umstände Einfluss. Dic von mir beobachteten Krystallisationen zeigen Folgendes:

4. Die Linschlüsse erscheinen unter denjenigen Flïchen, welche am energischsten wachsen; sie bäufen sich hauptsächlich unter den Combinatiouskinten derjenigen Fliichen an, welche verschiedene Wachsthumsgeschwindigkeit ljesilyen.

2. Unter den breilen Flïchen sind fast keine Einschlüsse, und bilden sie im Gegentheil einen trüben $A$ nwachskegel unter den verwachsenden und ver'wachsenen Flïchen. Mit anderen Worten: es befinden sich die Einschlüsse hauptsiichlich in jener Partic des Krystalles, wo ein freier Zufluss einer grossen Menge des übersättigenden Ueberschusses stattfand, wenn auch die Lösung schwach übersälligt war.

3. Von mehreren in einem (iefisse gleichzcitig wachsenden Keimen erhïlt man neben einander Krystalle mit Einschlüssen, und andere ohne solche.

Die Einschlüsse erscheinen manchmal bloss in einem Theile des durchsichtigen Krystalles als Folgen des intensiveren Wachsens nach einer Richtung. Die Pertubationen der Strüme finden also sogar in schwach übersätligter Lüsung statt, wenn der sich ausschcidende Ueberschuss sich unter die wachsenden Krystalle ungleichmüssig vertheilt. 1)er gleichmüssige Zulluss des Stofles wird durch eine gleichmässige Anordnung der Krystalle auf dem Boden des Gefässes erreicht. Selbstverständlich ist bei der polyëdrischen Form der Krystalle und bei ihrer Anisotropie das Erreichen ihrer gleichmüssigen Anordnung in der Ebene fast unmöglich. Deswegen wuchsen in meinen Versuchen zu gleicher Zeit durchsichtige und mit Einschlüssen überfüllte Krystalle, obgleich sie sich alle aus gleichen Keimen bildeten und unter gleichen Umstïnden (in cinem Gicliisse) wuchsen.

1) l. c. S. 460 . 
4. Irgend welche zufällige ürtliche Stürungen in der Lüsung rufen die Erscheinungen der Einschlüsse in den Krystallen hervor. Zu solchen Perturbationen gehört es auch, wenn einer der Krystalle sehr rasch wächst und deshalb besonders starke Ströme hervorruft (weil er als Keim ein unförmliches Bruchstück, ein Zwilling oder ein Aggregat war).

Was diese Beobachtung anbetrifft, so bleibt es uns übrig, Folgendes zu constatiren: Die in den Strümen durch einen Krystall hervorgerufenen Perturbationen wirken nicht nur auf ihn selbst, sondern auch auf die in der Nähe befindlichen Krystalle, wenn diese Perturbationen stark genug sind.

5. Die Anwendung kleiner Kryställchen als Keime, sogar in stark übersätligter Lösung, giebt mehr Garantie zur Erzielung homogener Kryṣtalle, als die $\Lambda$ nwendung grosser Keime in schwach übersättigter Lösung.

So erhielt ich in Neun-Literversuchen bei $1 \%$ übersättigendem Ueberschusse, aus zehn sehr kleinen Keimen von zwei Millimeter und weniger ganz homogene Krystalle. Zehn solcher Keime in fünf Liter mit $2 \%$ übersättigendem Ueberschusse gaben dasselbe Resultat. Wenn ich jedoch in sehr schwach übersättigter Lïsung (unter $1_{i 0}^{0}$ ) ziemlich grosse (gegen zwei Cubikcentimeter Volum), gut durchsichtige Irystalle legte, so erhielt ich Krystalle mit trüben Anwachskegeln unter den Würfelflïchen; diese Anwachskegel befanden sich bloss da nicht, wo in Folge der kleinen Entfernung zwischen den Krystallen die Würfelflächen nicht verwuchsen; das Wichtigste dabei ist die Erscheinung der Einschlüsse nicht nur ganz nahe an dem Keime, sondern auch ihre Bildung bis zum Ende des Versuches, wenn die Uebersättigung bis zum müglichen Minimum gefallen war $-d . h$. die $\Lambda$ nwachskegel durchdringen in den meisten Füllen die ganze Dicke der angewachsenen Kruste. Die meisten Einschlüsse unter den Würfelflächen erhielten diejenigen Krystalle, die auf den ()ktaëderflächen lagen; weniger Einschlüsse besassen die auf dem Würfel liegenden Krystalle, und jene, welche auf dem Dodekaëder lagen, erschienen vollständig durchsichtig. Durch Verminderung der Lücken zwischen den Krystallen machte ich diese Anwachskegel schwächer; es war mir aber unmüglich, sie vollständigg in den Versuchen mit grossen Keimen zu vermeiden.

Wenn man also grosse homogene Krystalle erhalten will, muss man टine grosse Menge nicht stark übersältigter Lüsung nehmen und darin eine bedeutende Zahl müglichst kleiner Keime weiter züchten; die Lücken zwischen den Keimen dürfen nicht zu breit sein.

6. Wenn sich aus der Lüsung, in welche die Keime gelegt wurden, Krystalle selbständig unabhängig von den Keimen ausscheiden, so enthalten diese secundïren Krystalle grüsstentheils keine Einschlüsse; wenn sie jedoch Einschlüsse haben, so ist die Menge derselben sehr unbedeutend und sie sind ganz regelmiissig angeordnet, respective gegen dic Combinationskanten streng orientirt. 
In den Versuchen über das Wachsthum der Alaunkrystalle auf den fünf Millimeter grossen Keimen aus der Lösung, in welcher gegen 2,5\% ursprünglicher übersittligender Ucberschuss vorhanden war, wurden die in die Lüsung gelegten Keime im Laufe von 24 Stunden vom Anfange des Versuches viel grösser und blieben dabei durchsichtig. Zu dieser Zeit waren die Flächen $\{100\}$ und $\{110\}$ schon ziemlich breit. Erst am nächsten Tage fingen fast alle Krystalle an mit liinschlüssen zu wachsen (einige darunter blieben durchsichtig) und am vierten oder fünften Tage des Versuches wuchs schon eine durchsichtige Kruste auf derjenigen Zone, welche Einschlüsse hatte.

Ausser der ersten Generation von ausgeschiedenen Krystallen oder den weitergezüchteten Keimen erscheint eine zweite Generation von Krystallen zur Zeit, wenn die Concentrationsströme das Maximum ihrer Stärke crreichen; wenn die Ströme schwïcher werden, erscheint eine dritte Generation und zuweilen auch eine vierte. Meistentheils sind die Krystalle dieser secundären Bildungen tadellos, und die Einschlüsse, die sich darin manchmal befinden, sind sehr unbedeutend und ganz regelmässig angeordnet. Die Figur 2, S. 54 stellt nämlich den Durchschnitt des Anwachskegels von einem derartigen Kryställchen dar. Solche Krystalle sind meistentheils isosymmetrisch, die Flïchen aller drei Formen sind fast gleichmüssig entwickelt, haben keine Vicinalflächen und rufen besonders schwache Concentrationsstrüme hervor, obwohl sie sich manchmal aus ziemlich stark übersüttigter Lüsung ausscheiden und von den daneben wachsenden grossen primären Krystallen sich dicke Bündel von deutlichen Concentrationsstrümen crheben.

Endlich, was die Umstïnde der Entstehung einschlussfreier Krystalle anbetrifft, kann ich folgenden recht interessanten Versuch anführen.

Ein grosser wasserklarer Kaliumaluminium-Alaunkrystall wurde im Laufe von 24. Stunden in seiner eigenen Lösung in einem rotirenden Gefässe weiter gezüchtet. Die Bedingungen waren folgende: $350 \mathrm{ccm}$ Lösung, Uebersättigung 2\%, Krystallisirgefüss acht Centimeter hoch, der Diameter des Bodens 7,5 Centimeter, Temperatur $+15^{\circ} \mathrm{C}$., zwei Umdrehungen in einer Minute, die Breite von (110) vor dem Versuche fünf Millimeter, von (001) sieben Millimeter. Während des Versuches waren starke Concentrationsstrüme zu sehen. Nach dem Versuche war der Krystall noch immer durchsichtig, und zwischen der angewachsenen Kruste und dem Kern war kein Unterschied zu bemerken. Wie jedoch früher angegeben wurde, wuchsen grosse Keime in bewegungslosen Krystallisirgefässen sogar in $0,70_{i}^{\circ}$ Uebersättigung weiter mit Einschlüssen. Der in der Lüsung sich bewegende Krystall vermischte offenbar die Schlieren von verschiedener Dichte, was das Entstehen der Perturbationen in den Strümen an den Kanten hinderte. 


\section{§ 8. Allgemeine Uebersicht und einige kritische Bemerkungen.}

Bei dem Krystallisationsprocesse eines löslichen Salzes in seiner Mutterlauge sehen wir von dem Momente an, in welchem sich auf dem Boden des Gefässes ein winziges Kryställchen ausgeschieden hatte, dass an diesem Processe bloss diejenige Menge des aufgelösten Stoffes theilnimmt, welche die Lösung bei der bestimmten Temperatur übersättigt. Einige Forscher (wie L. Wulff) behaupten, dass das Lösungsmittel während der Krystallisation >frei « wird. Solche Ansicht steht im Widerspruche mit dem Begriffe der "gesättigten Lösung * ${ }^{1}$ ), denn so wird eine Flüssigkeit genannt, in welcher der Krystall weder wächst, noch sich auflüst; eine solche Lösung erhält man entweder durch das so lange fortgesetzte Auflösen des Stofles, bis dieser aufhört sich bei der angegebenen Temperatur zu lösen, oder durch Krystallisation des Ueberschusses des aufgelüsten Stofles bis zu seiner vollstïndigen Ausscheidung. Die sättigende Menge des Stoffes ist durch das Lösungsmittel bei einer gewissen Temperatur im bestimmten Verhältnisse gebunden, und ohne die Temperatur zu erniedrigen, oder ohne das Iüsungsmittel durch Abdampfen zu entfernen, kann man diesen sättigenden Stoff nicht zur Ausscheidung bringen. Dic gesättigte Lösung verhält sich gegen den Krystall indifferent. Sie bildet bloss das Medium, welches den wachsenden Krystallen den aus der Lüsung sich ausscheidenden Stofl zuführt und ihn zwischen die Krystalle vertheilt. Die Concentration der Lösung wührend der Krystallisation kann in keinem Falle unter den Süttigungspunkt fallen, wenn die Temperatur nicht steigt.

Auf dem Krystalle lagert sich der die Lösung übersättigende Ueberschuss $a b$, infolge dessen erheben sich vom Krystalle Ströme der Lüsung, welche in Folge des Wachsens des Krystalles weniger übersättigt wurde. Diejenigen Ströme, welche vom Krystalle nach oben steigen, rufen unterhalb fliessende Ströme, die zum Krystall hin gerichtet sind, hervor; letztere sind stärker übersättigt als die aufsteigenden und bringen neue Mengen des Stoffes herbei. Die Ströme erheben sich inzwischen, verdünnen zum Theil die Lösung unterwegs, erreichen die Oberflüche der Flüssigkeit, werden reflectirt 2), pflanzen sich in der oberen Schicht der Lösung fort und verdünnen diese Schicht. Diese Verdünnung durchdringt immer tiefer die Lüsung vermittelst der Dilfusion, und die niedersteigenden Strüme, welche als Resultat der nach oben steigenden erscheinen, führen den Krystallen immer weniger Stoff zu.

Die allgemeine Geschwindigkeit des Wachsens des ganzen Krystalles nimmt mit der Vergrösserung der Oberfläche des Krystalles zu und füllt mit der Erniedrigung der Uebersüttigung. Im Allgemeinen wächst der

1) Siehe darüber G. Wulff I. c. S. 449, 454, 461, 467, 468 .

2) Siehe die Arbeit von G. Wulff l. c. Tal. VII. 
Krystall anfangs sehr langsam; wenn er eine gewisse Grüsse erreicht, so ruft er immer energischere Ströme hervor, und sein Wachsthum nimmt sehr rasch zu. Nachdem die Concentrationsströme cin gewisses Maximum der Geschwindigkeit erreicht haben, bemerkt man wieder eine Verkleinerung der Wachsthumsgeschwindigkeit des Krystalles, die Ströme und der Zuwachs des Krystalles werden schwächer, die Ströme verkürzen sich und verschwinden endlich vollstïndig. Eine scheinbare Wachsthumsgrenze tritt ein. Die Lüsung ist inzwischen noch übersättigt: mit dem festen Stoffe geschüttelt odcr berührt, scheidet die Lösung einen feinen krystallinischen Niederschlag des übersüttigenden Ueberschusses aus ${ }^{1}$ ).

Wenn die Strömo ihre volle Stärke erreicht haben, erscheinen secundäre Generationen von Krystallen. Das bedeutet aber nicht, wie Manche meinten (Retgers), dass die primären Krystalle nicht mehr zu wachsen im Stande wären. Im Gegentheil, man sieht unmittelbar, wie sie manchmal noch energisch wachsen und starke Ströme hervorrufen. Die Ausscheidung. dieser secundïren Krystalle führt vielmehr zu dem Gedanken, dass in der Lösung, hauptsächlich wenn sie in grosser Menge vorhanden ist, ein Moment eintritt, wo die allgemeine Circulation in der Lösung in einzelne Systeme zerfaillt. In diesem Momente entsteht eine besondere Schwierigkeit für die Uebertragung der gesammten Uebersïttigung zu allen anwesenden Krystallen, und deswegen scheidet sich zu dieser Zeit der übersüttigende Ueberschuss selbständig aus. Alle diese secundären Krystalle sind immer homogen und crreichten in meinen Versuchen über zwei Cubikcentimeter Grösse. Sie wachsen gewöhnlich sehr glcichmässig und ruhig, obgleich, wie ich vorher bemerkte, von den grossen, daneben wachsenden, aus den Keimen entstandenen Krystallen sich starke Ströme erheben.

Man kann also schliessen: Die Geschwindigkeit des Wachsthums des ganzen Krystalles nimmt zu mit der Vergrösserung seiner Oberfläche.

Alles in diesem Kapitel Gesagte scheint mir schon lange bekannt und ganz elementar zu sein. Indessen gicbt es Arbeiten, in welchen andere Meinungen ausgesprochen wurden. Hr. Ludwig Wulff schreibt nämlich 2): \)ie Lösungen bei den meisten Versuchen, Krystalle zu ziehen, sind nicht durch die ganze Menge gleich concentrirt, besonders nicht bei schwankenden Temperaturen, sondern am Boden ist die Lüsung ein wenig concentrirter. Je grösser der Krystall wird, in desto hühere Schichten ragt derselbe hinein. Treten nun Unterconcentrationen ein, so sind die oberen Partien der grossen Krystalle denen sehr ausgesetzt und es lüsen sich leicht diesclben unter Aetzung oder Abrundung. Die abgelösten Krystalltheile

1) Erklärung s. G. Wulff l. c. S. 462.

2) X. Jahrl. f. Min. etc. $1896,2,434$. 
geben zur Bildung concentrirterer Lösungstheile Veranlassung, die zu Boden sinken, wo sich dann leicht Ueberconcentration und Neubildung von Krystallen einstellen. Sind erst kleine Krystalle vorhanden, so befinden sich diese in günstigerer Lage als die grossen Krystalle, aber nicht, weil diese nicht mehr hälten wachsen können, sondern weil ihnen die Zufuhr von Substanz abging. « Der Autor bemerkte also die von 0. Lehmann beschriebenen Concentrationsströme nicht. Aus seinen citirten Worten könnte man schliessen, dass unmittelbar bei der Krystalloberfläche sich ein zu Boden sinkender Strom befindet, was unrichtig ist; denn nehen dem, sogar aus schwach übersüttigter Lüsung wachsenden Krystalle sehen wir nach oben steigende Ströme, die ihn so eng umhüllen, dass sie keine Müglichkeit der Existenz von anderen Strömen in der Nähe des Krystalles bieten. IIr. L. Wulff behauptet, dass die Lösung während der Krystallisation bei constanter Temperatur ungesättigt werden kann. Nun dürfte aber doch unzweifelhaft bekannt sein, dass Krystalle sich bloss aus übersättigter Lösung ausscheiden; die Uebersättigung der Lüsung füllt je nach dem Wachsthume der Krystalle, und die Deconcentrirungsgrenze der übersättigten Lösung ist der Süttigungszustand. Diese Grenze überschreitet die Deconcentrirung nicht. Nach den Worten von Hrn. L. Wulff findet in der Lösung gleichzeitig das Wachsthum und die Auflösung des Krystalles stalt ${ }^{1}$ ). Indem ich die theoretische Seite dieser Frage übergehe, welche der Behauptung von Hrn. L. Wulff voliständig widerspricht, kann ich sagen, dass ich öfters, und zwar mannigfache verschiedene Substanzen, bei constanter Temperatur aus einer dünnen Schicht der Lüsung krystallisirte und die Krystallisation bis zu derjenigen Grenze brachte, welche zu erreichen müglich war; man controlirt dies durch Beobachtung der Ströme und durch das Gewicht der Krystalle nach dem Versuche. Meine Krystalle wuchsen manchmal so weit aus, dass sie die Oberfläche der Lösung berührten, und dic Lüsung, nachdem die Krystalle herausgenommen wurden, schied weitcr keinen Niederschlag aus; man konnte aber weder mit blossem $\Lambda$ uge, noch unler dem Mikroskope Aetzfiguren an den Krystallen finden, und ihre Kanten und Ecken waren nicht abgerundet.

Auf S. 135 der citirten $\Lambda$ bhandlung von L. Wulff lesen wir: „So können sich sehr wohl die Fremdkürper auf der Oberfläche so ansammeln, dass die Diffusionserscheinungen an der Oberfläche dadurch gehemmt werden. * Meine früher angegebenen Beobachtungen über die Trübungen in der Lösung und von ihrer Anordnung auf dem Krystalle widerlegen diese Meinung des Autors. Wenn man den mit der 'Trübung bedeckten Krystall von der Seite betrachtet, sieht man, dass die Leinwand- und Lüschpapierfasern und dic Flocken des basischen Salzes fast senkrecht über der Kry-

1) Siehe G. Wulff l. c. S. 468 . O. Lehmannn, Molekularphysik 1, 209. 
Einige Beobachtungen über das Wachsth. der Kaliumaluminium-Alaunkrystalle. 61

stalloberfläche schweben und sie kaum berühren. Deswegen wachsen auch diese Körperchen nicht in den Krystall hinein, sie steigen gleichzeitig mit dem Wachsen der Fläche (111) nach oben, denn sie liegen nicht auf der Oberfläche des Krystalles: sie werden durch die Concentrationsstrüme von der Oberfläche auf eine gewisse Entfernung hin fortgerissen. Unter diesen Umständen kann also die Diffusion durch diese Fremdkörper nicht gehemmt werden. Da die Lösungen sogar durch Membranen diffundiren, so können diese Fremdkörper die Diffusion auch dann nicht hemmen, wenn die Strüme verschwinden und die Trübung sich auf der Krystalloberfläche absetzt.

Hr. L. Wulff sagt endlich Folgendes ${ }^{1}$ ): „0. Lehmann hat ja durch seine Untersuchungen gezeigt, dass die Krystalle um sich einen Ilof von minder concentrirter Lösung haben. Bildet sich also ein Krystall, so entsteht er in einer Flüssigkeitspartie, die stärker concentrirt ist, als später seine unmittelbare Ungebung. Es muss erst durch den Krystall sovie Substanz an sich genommen werden, bis der Hof ausgebildet ist; erst wenn die IIofbildung vollendet ist, beginnt das langsame Wachsthum. So haben wir im Wachsthume der Krystalle zwei Stadien zu unterscheiden. Bei sehr kleinen Dimensionen nimmt die Wachsthumsgeschwindigkeit schnell $a b$, bis die Hofbildung vollendet ist, dann steigt sie langsain mit der Grüsse der Krystalle. Dies gilt natürlich nur für Normalverhältnisse; in überconcentrirter Lösung können grosse Krystalle ja auch noch schnell wachsen."

Was » Krystallisationshof « bedeutet, wissen wir aus der $\Lambda$ rbeit IIrn. G. Wulff's l. c. S. 460.

Die Wachsthumsgeschwindigkeit des kleinen Krystalles nimmt nach den Worten von $\mathrm{L}$. Wulff schnell ab und dann wieder lingsam zu. In Wirklichkeit jedoch geschieht es gerade umgekehrt: die Wachsthumsgeschwindigkeit nimmt nur zu von dem Momente an, wenn der Krystall entsteht, bis die Concentrationsstrüme ihre volle Stärke erreicht haben; später nimmt die Wachsthumsgeschwindigkeit ab, je nach der Verminderung der Ströme, indem sich alsdann nur noch die leste des übersättigenden Ueberschusses ausscheiden.

Endlich ist nach der Meinung von Hrn. L. Wulff das Wachsthum des Krystalles in der übersättigten Lösung eine »anormale Erscheinung. Wann kann man also das Wachsthum des Krystalles »normal « nennen, und in welchen Lösungen erhielt der Autor seine Krystalle?

1) S. 136. 